POS $\quad$ PROCEEDINGS

\title{
Neutrons from multifragmentation reactions
}

\author{
W. TRAUTMANN* \\ GSI Helmholtzzentrum Darmstadt, Germany \\ E-mail: w.trautmannegsi.de
}

\section{A.S. BOTVINA}

INR Moscow, Russia

\section{J. BRZYCHCZYK}

Jagiellonian University, Kraków, Poland

\section{N. BUYUKCIZMECI}

Selçuk University, Konya, Turkey

\section{I.N. MISHUSTIN}

FIAS, Goethe Universität, Frankfurt, Germany

\section{P. PAWŁOWSKI}

IFJ-PAN, Kraków, Poland

\section{ALADIN2000 COLLABORATION}

The neutron emission in the fragmentation of stable and radioactive $\mathrm{Sn}$ and La projectiles of 600 $\mathrm{MeV}$ per nucleon has been studied with the Large Neutron Detector LAND coupled to the ALADIN forward spectrometer at SIS. A cluster-recognition algorithm is used to identify individual particles within the hit distributions registered with LAND. The obtained momentum distributions are extrapolated over the full phase space occupied by the neutrons from the projectile-spectator source. The mean multiplicities of spectator neutrons reach values of up to 12 and depend strongly on the isotopic composition of the projectile. An effective source temperature of $T \approx 3-4 \mathrm{MeV}$ is deduced from the transverse momentum distributions.

For the interpretation of the data, calculations with the Statistical Multifragmentation Model for a properly chosen ensemble of excited sources were performed. The possible modification of the liquid-drop parameters of the fragment description in the hot environment is studied, and a significant reduction of the symmetry-term coefficient is found necessary to simultaneously reproduce the neutron multiplicities and the mean neutron-to-proton ratios $<N>/ Z$ of $Z \leq 10$ fragments. Because of the similarity of the freeze-out conditions with those encountered in supernova scenarios, this is of astrophysical interest.

XLIX International Winter Meeting on Nuclear Physics

24-28 January 2011

BORMIO, Italy

\footnotetext{
* Speaker.
} 


\section{Introduction}

Neutron emission is an efficient means of cooling excited nuclei produced in nuclear reactions. For this reason, e.g., (heavy ion,xn) reactions have been and still are a standard tool for the spectroscopy of nuclei at high spin. Heavy projectiles will transfer large amounts of energy and angular momentum to the formed compound nuclei. Neutron evaporation then removes most of the energy but very little of the angular momentum, so that high-spin states close to the yrast line will be fed by the subsequent gamma decay. Superdeformed bands at the highest spins observed have been discovered in this way [1]. By emitting neutrons the product nuclei also change their isotopic composition, permitting high-spin spectroscopy deep into the neutron-poor region of the chart of nuclides. Neutron emission is thus important in the energetic evolution of heavy-ion reactions and, at the same time, related to isotopic phenomena.

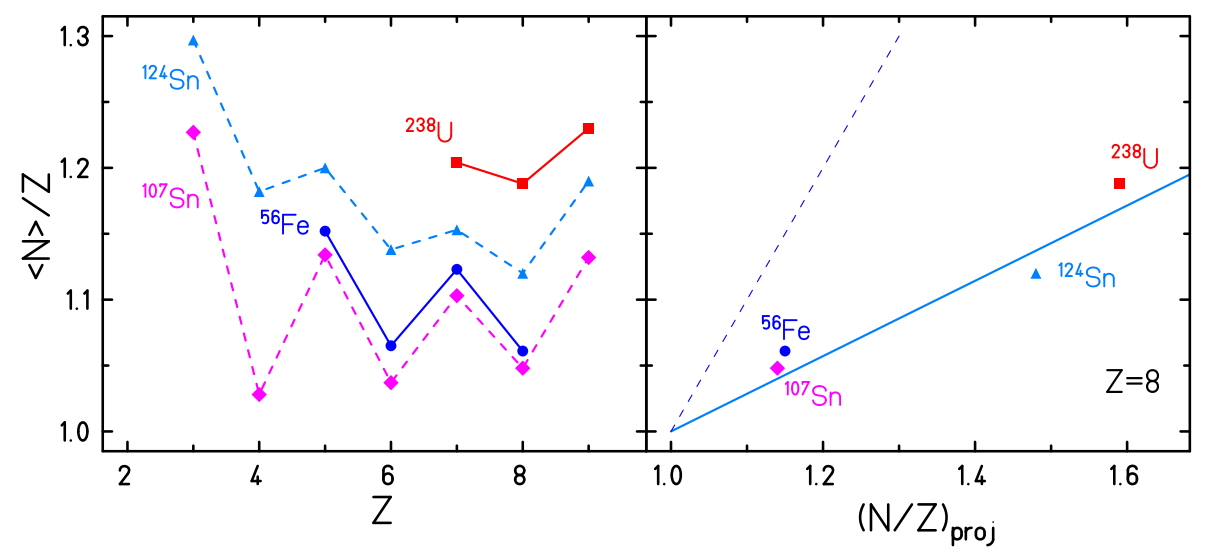

Figure 1: Inclusive mean values $<N>/ Z$ of light fragments with $3 \leq Z \leq 9$ produced in the fragmentation of ${ }^{107,124} \mathrm{Sn}\left(600 \mathrm{MeV} /\right.$ nucleon, ALADIN), ${ }^{56} \mathrm{Fe}$ and ${ }^{238} \mathrm{U}$ (both $1 \mathrm{GeV} /$ nucleon, FRS) as a function of the fragment $Z$ (left panel). The values for $Z=4$ have been corrected for the missing yield of unstable ${ }^{8} \mathrm{Be}$ by including an estimate for it obtained from a smooth interpolation over the identified yields of ${ }^{7,9-11} \mathrm{Be}$. This correction makes the systematic odd-even variation more clearly visible for the neutron rich case. The right panel shows the results for $Z=8$ as a function of the $N / Z$ value of the projectile. The lines represent the trend of the data (full line) and $<N>/ Z=(N / Z)_{\text {proj }}$ (dashed); from Ref. [8].

Long evaporation cascades will include charged particle emissions which will eventually compete with neutron emission in neutron-poor nuclei. This has led to the concept of a universal evaporation attractor line (or fragmentation corridor) for the location of final residues in the plane of neutron number $N$ vs. atomic number $Z$. Predictions have been calculated with statistical models or were empirically deduced from experimental data $[2,3,4]$. They are very useful for a wide range of reactions but deviations exist. In multifragmentation reactions, observed at excitation energies above a threshold of about $3 \mathrm{MeV}$ per nucleon, the neutron content of produced fragments depends significantly on the $N / Z$ ratio of the reaction system. This is illustrated in Fig. 1 with data measured with the ALADIN forward spectrometer and the FRS fragment separator at GSI [5, 6, 7].

Multifragmentation is a rapid breakup of the highly excited reaction system and associated with a copious production of nuclear fragments in addition to neutrons and light charged particles (for a review see, e.g., Ref. [9]). Statistical models have been found very useful for describing the partitioning of the excited systems $[10,11,12,13,14,15,16,17]$. They have also been used 
to explore possible modifications of fragment properties in the hot environment of the freeze-out state. This is of interest because the temperatures and lower than normal densities at freeze-out are similar to conditions encountered in supernova scenarios $[18,19]$.

As shown very recently for the case of multifragmentation of relativistic projectiles [20], the observed neutron richness of the produced intermediate-mass fragments requires a significant reduction of the symmetry term in the liquid-drop description used for them in the Statistical Multifragmentation Model (SMM, Ref. [11]). With more neutrons bound in fragments, the multiplicity of free neutrons should be lowered, thereby possibly offering an additional possibility for exploring fragment properties at freeze-out. This expectation can be tested since the Large Area Neutron Detector LAND [21] was used in these experiments to detect neutrons from the breakup of the projectile and subsequent decays. Results from this study will be reported here.

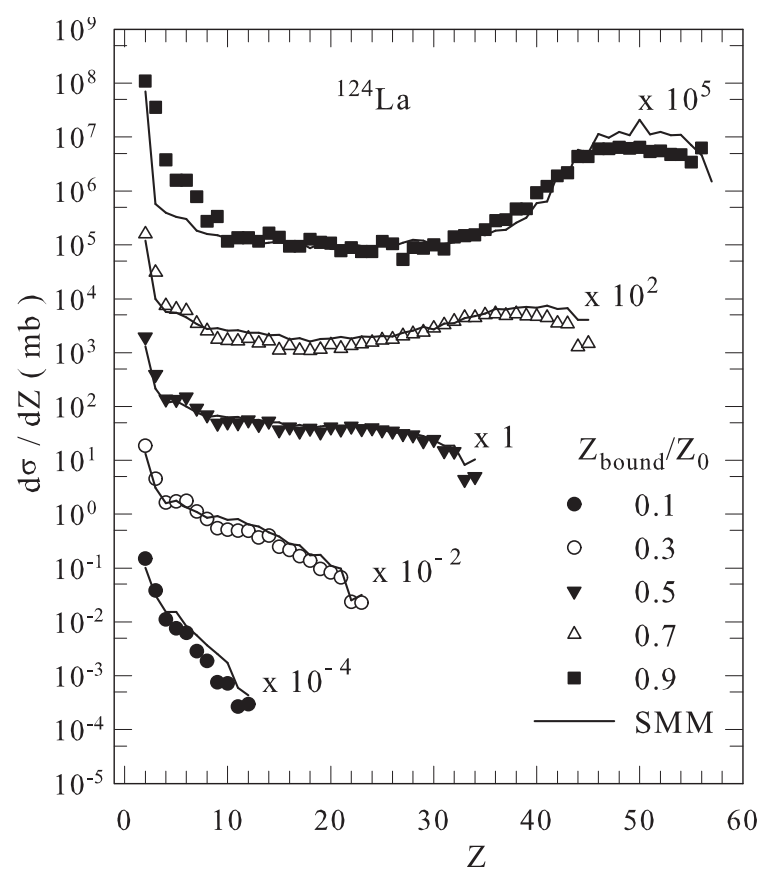

Figure 2: Experimental cross sections $\mathrm{d} \sigma / \mathrm{d} Z$ for the fragment production following collisions of ${ }^{124} \mathrm{La}$ projectiles sorted into five intervals of $Z_{\text {bound }} / Z_{0}$ with centers as indicated and width 0.2 (symbols) in comparison with normalized SMM calculations (lines, $Z_{0}=Z_{\text {proj }}$ ). Different scale factors were used for displaying the cross sections as indicated (from Ref. [20]).

In multifragmentation experiments, the detection of neutrons has also been essential for investigating the transfer of energy. In previous ALADIN experiments, the excitation energy of the produced spectator systems was determined by using in particular also the neutron energies and multiplicities measured with the LAND detector [22]. Multifragmentation experiments including the measurement of neutrons, although difficult because of the different techniques required for simultaneously detecting neutral and charged particles, have been performed at several laboratories $[23,24,25,26,27]$. The problems and successes encountered in the calorimetry of multifragmenting systems have recently been reviewed [28]. 


\section{Isospin dependent multifragmentation}

Isotopic effects in projectile multi-fragmentation at relativistic energies were studied with experiment S254 of the ALADIN collaboration, conducted in 2003 at the SIS heavy-ion synchrotron [7, 20]. Besides stable ${ }^{124} \mathrm{Sn}$ beams, neutron-poor secondary ${ }^{107} \mathrm{Sn}$ and ${ }^{124} \mathrm{La}$ beams provided by the FRS fragment separator, all with $600 \mathrm{MeV} /$ nucleon incident energy, were used in order to explore a wide range of isotopic compositions. For the interpretation of the data, calculations with the Statistical Multifragmentation Model [11] for a properly chosen ensemble of excited sources, adapted to the participant-spectator scenario at relativistic energies, were performed. The ensemble parameters were chosen so as to best reproduce the $Z$ spectra and correlations of the observed fragments.

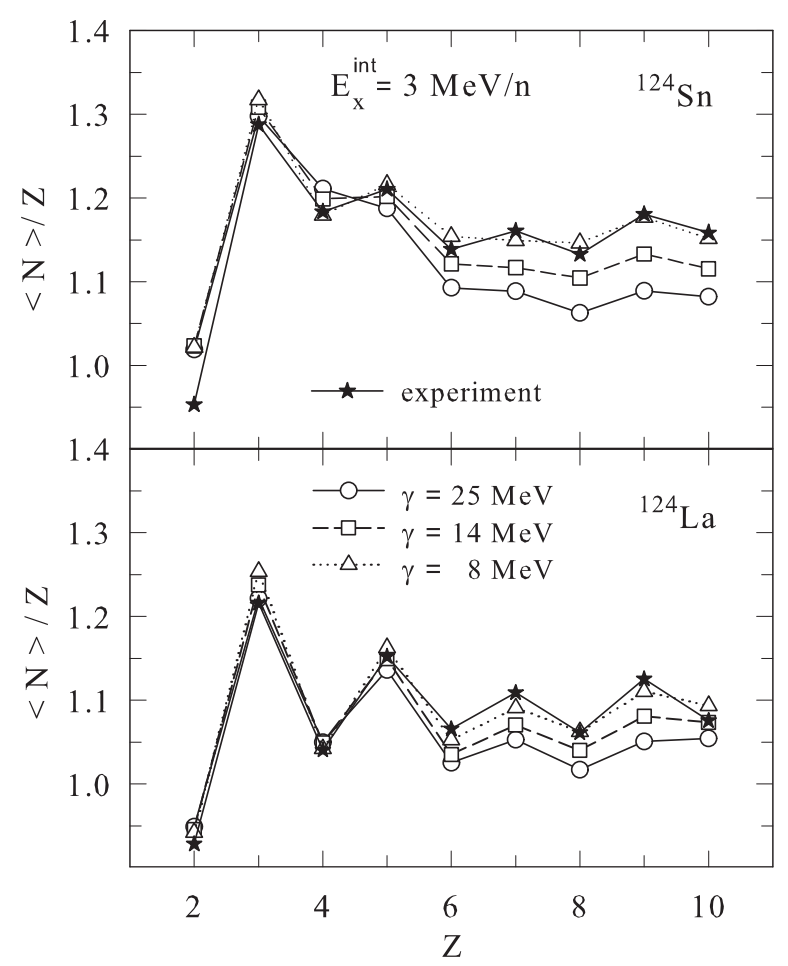

Figure 3: Mean neutron-to-proton ratio $\langle N\rangle / Z$ of fragments produced in the fragmentation of ${ }^{124} \mathrm{Sn}$ (top panel) and ${ }^{124} \mathrm{La}$ (bottom panel) projectiles in the range $0.4 \leq Z_{\mathrm{bound}} / Z_{\mathrm{proj}}<0.6$. The experimental results (stars) are compared with SMM calculations using the three indicated values of the symmetry-term coefficient $\gamma$ and $E_{x}^{\text {int }}=3 \mathrm{MeV} /$ nucleon as the start value of the interpolation interval for the secondary-decay stage of the calculations. With this choice, $\gamma=8 \mathrm{MeV}$ (triangles) gives the best agreement with the data.

As an example of the good description achieved, the measured cross sections $\mathrm{d} \sigma / \mathrm{d} Z$ for fragment production in reactions initiated by ${ }^{124}$ La projectiles are shown in Fig. 2. The data have been sorted into five bins of the reduced bound charge $Z_{\text {bound }} / Z_{\text {proj }}$ of width 0.2 , spanning the range up to $Z_{\text {bound }}=Z_{\text {proj }}\left(Z_{\text {bound }}=\Sigma Z_{i}\right.$ for $\left.Z_{i} \geq 2\right)$. The charge distribution evolves from a so-called 'U-shaped' distribution, with domination of evaporation of the compound nucleus and asymmetric binary decays, to a rapidly dropping exponential distribution. This evolution is a well-known characteristic feature and has been related to the nuclear liquid-gas phase transition [29, 30, 31]. 
The successful description of charge observables provides the basis for the investigation of the isotope distributions and the information contained therein. As shown in more detail in Ref. [20], it is mostly the symmetry-term coefficient $\gamma$ which influences the isotopic composition of fragments. Model calculations performed for three values of $\gamma=8,14$, and $25 \mathrm{MeV}$ are shown in Fig. 3. The mean neutron-to-proton ratio $\langle N\rangle / Z$ increases with decreasing $\gamma$, most noticeably for $Z \geq 7$. A reduced symmetry term leads to larger widths of the initial isotope distributions at freeze-out and to a reduced driving force toward the line of stability during subsequent decays. This effect is larger for the more neutron-rich system ${ }^{124} \mathrm{Sn}$. At $Z \leq 6$, the mean neutron-to-proton ratios exhibit the strong odd-even effects, typical for this type of reaction (Fig. 1).

While the fragment properties may be modified at freeze-out and during the first deexcitation steps, as the hot fragments are still surrounded by other species, the standard properties must be restored at the end of the evaporation cascade. In the calculations, a linear interpolation between these two limiting cases has been introduced in the interval of excitation energies between zero and a value $E_{x}^{\text {int }}$ which has been varied between 1 and $3 \mathrm{MeV} /$ nucleon. Energy and momentum conservation are observed throughout this process. The results shown in Fig. 3 were obtained for $E_{x}^{\text {int }}=3 \mathrm{MeV} /$ nucleon. In this case, the best agreement with the experimental results is obtained with $\gamma=8 \mathrm{MeV}$, while $\gamma=14 \mathrm{MeV}$ appears to be the better choice if the interpolation towards the standard $\gamma=25 \mathrm{MeV}$ is delayed until the excitation energy is at or below $1 \mathrm{MeV} /$ nucleon [20].

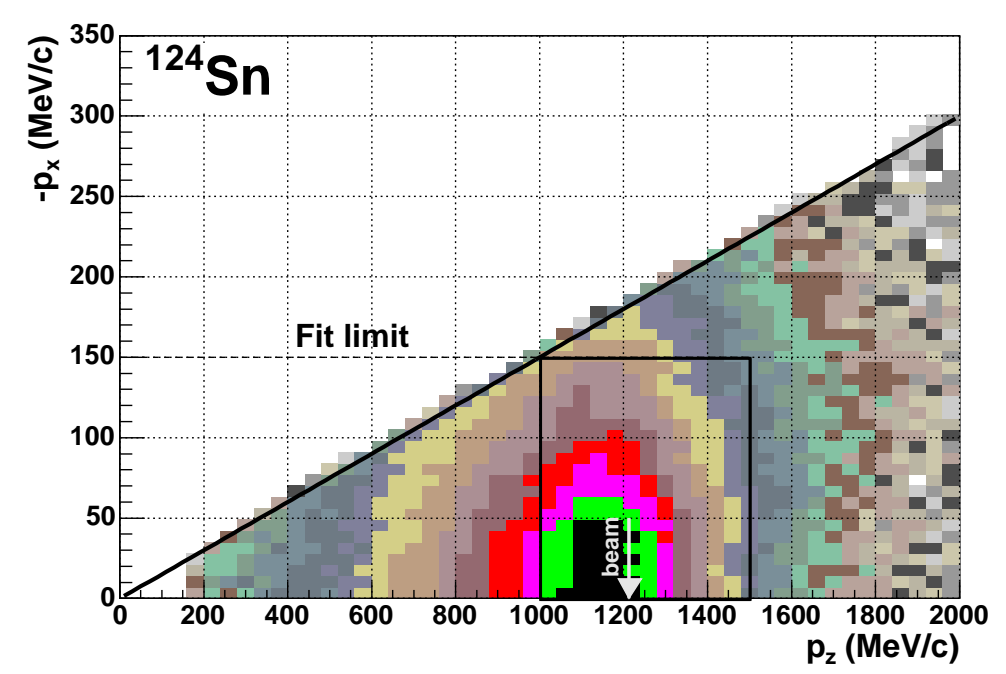

Figure 4: Distribution of identified neutrons in the plane of transverse momentum $p_{x}$ versus longitudinal momentum $p_{z}$ for the fragmentation of ${ }^{124} \mathrm{Sn}$ projectiles in collisions with natural Sn targets at an incident energy of $600 \mathrm{MeV} / n u c l e o n$. The full line indicates the acceptance cut in the analysis. The thin rectangle indicates the two-dimensional interval $\left|p_{x}\right| \leq 150 \mathrm{MeV} / \mathrm{c}$ and $1000 \leq p_{z} \leq 1500 \mathrm{MeV} / \mathrm{c}$ used for the determination of the source temperature.

A similar result was obtained from the isoscaling analysis performed with the same data and discussed in Ref. [20]. The isotopically resolved fragment yields provide evidence for a reduced symmetry energy in the hot environment at freeze-out, in accordance with previous findings [32, 33]. Similar observations were made in other reaction studies performed at intermediate and relativistic energies [34, 35, 36, 37]. 


\section{Neutron detection and analysis}

The Large-Area-Neutron-Detector LAND is a $2 \times 2 \times 1 \mathrm{~m}^{3}$ calorimeter consisting of in total 200 slabs of interleaved iron and plastic strips viewed by photomultiplier tubes at both ends [21]. In the ALADIN experiments, the detector was positioned approximately $10 \mathrm{~m}$ downstream from the target and slightly asymmetrically with respect to the incoming beam direction, so as to allow the deflected beam passing along it on one side [38]. The angular acceptance of $-9.8^{\circ}$ to $1.2^{\circ}$ in horizontal and $\pm 5.6^{\circ}$ in vertical directions corresponded to a geometrical acceptance in perpendicular momenta $p_{x}$ from approximately $-210 \mathrm{MeV} / \mathrm{c}$ to $25 \mathrm{MeV} / \mathrm{c}$ and $\left|p_{y}\right| \leq 120 \mathrm{MeV} / \mathrm{c}$ for neutrons with a kinetic energy of $600 \mathrm{MeV}$. To avoid edge effects, the acceptance was reduced in the analysis. A veto wall of 5-mm-thick plastic scintillators in front of the detector permitted the distinction of neutral and charged particles.

In the analysis, a cluster-recognition algorithm is used to identify individual particles within the registered hit distributions. The mean number of hits belonging to an observed neutron event was found to be about 1.5. Particle momenta were determined from the neutron time-of-flight measured with a resolution of $\Delta t \approx 550 \mathrm{ps}$ (FWHM). For the case of ${ }^{124} \mathrm{Sn}$ projectiles, the obtained distribution of neutron events in the plane of transverse momentum $p_{x}$ versus longitudinal momentum $p_{z}$ is shown in Fig. 4. The source of neutrons emitted from the projectile spectator is clearly visible, centered near the projectile momentum $p_{\text {proj }}=1216 \mathrm{MeV} / \mathrm{c} /$ nucleon.

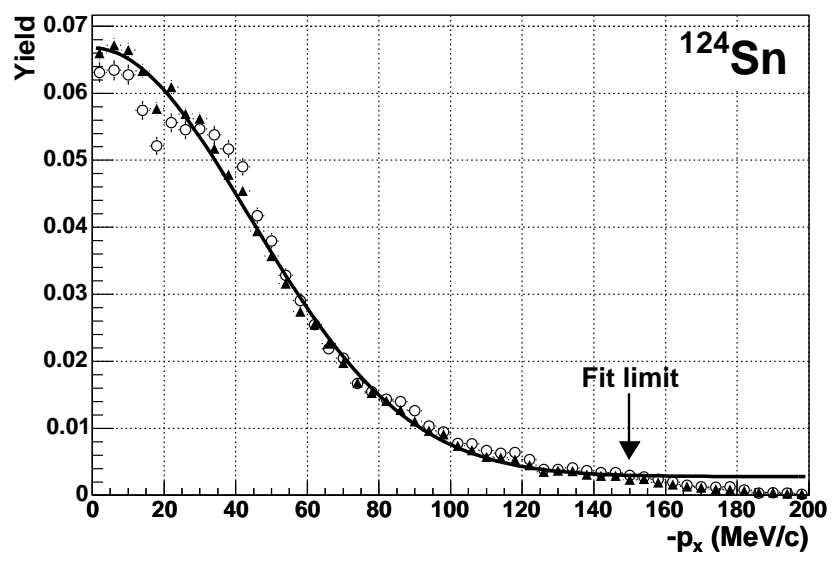

Figure 5: Distribution of transverse momentum $p_{x}$ for neutrons from the fragmentation of ${ }^{124} \mathrm{Sn}$ projectiles in collisions with natural Sn targets at an incident energy of $600 \mathrm{MeV} /$ nucleon, selected with the condition $Z_{\text {bound }} \geq 45$. The open circles and the filled triangles represent the yield of primary hits within a neutron cluster and the yields of all hits, respectively. The solid line shows the result of a fit with a Gaussian distribution superimposed on a constant background.

To determine neutron multiplicities of the spectator source, several steps had to be taken. The partial acceptance of LAND made it necessary to extrapolate from the kinematic regime covered by the detector to larger transverse momenta. This was done with Gaussian functions in $p_{x}$ and $p_{y}$ which were found to describe well the measured spectra (Fig. 5). Effective temperatures deduced from the distribution widths are close to $T=4 \mathrm{MeV}$ for $Z_{\text {bound }} \leq 20$ and smoothly decrease to $T \approx 2.5 \mathrm{MeV}$ at $Z_{\text {bound }} / Z_{\text {proj }} \approx 1$. No significant difference was observed between the tempera- 
tures of the neutron-rich and neutron-poor reaction systems, consistent with the result for isotope temperatures deduced from the yields of fragments and light charged particles [7].

The efficiency of LAND in this experiment for neutrons of about $600 \mathrm{MeV}$ was determined with a hit-rejection algorithm and also from the probability for zero hits obtained by fitting the measured hit number distributions of identified neutron events with Poissonian functions. Comparable results were obtained with the two methods, and a global efficiency value $\eta=0.73$ was adopted. The resulting multiplicities of the spectator neutron source are shown in Fig. 6. They clearly reflect the neutron richness of the system, exhibiting maxima of $M_{n} \approx 12$ for ${ }^{124} \mathrm{Sn}$ and $M_{n} \approx 9$ for ${ }^{124} \mathrm{La}$ at values of $Z_{\text {bound }} / Z_{\text {proj }} \approx 0.7$. They increase rapidly with increasing excitation energy in the regime of residue production $\left(Z_{\text {bound }} / Z_{\text {proj }}>0.8\right)$ and drop more smoothly from their maxima to smaller values at small $Z_{\text {bound. }}$.

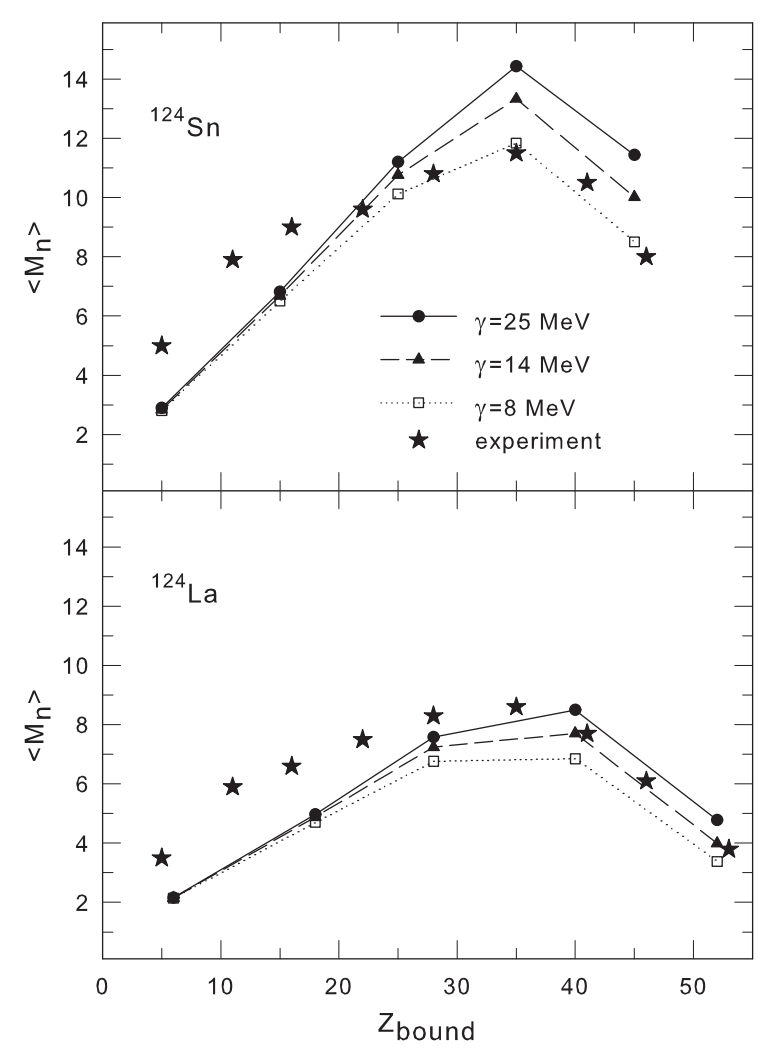

Figure 6: Mean neutron multiplicity of the spectator sources produced in the fragmentation of ${ }^{124} \mathrm{Sn}$ (top panel) and ${ }^{124} \mathrm{La}$ (bottom panel) as a function of $Z_{\text {bound }}$. The experimental data are represented by the stars. The results of the SMM ensemble calculations are shown for three values of the symmetry-term coefficient $\gamma$ and represented by the symbols and lines as indicated.

\section{Neutrons and the symmetry energy}

Predictions for the multiplicity of neutrons from the spectator decay obtained with the Statistical Multifragmentation Model are shown in Fig. 6. They were calculated with the ensemble and liquid-drop parameters previously determined in the study of fragment production in these re- 
actions [20]. As expected, the neutron multiplicity is sensitive to the symmetry term coefficient used in the calculations. A reduction of the symmetry term causes the neutron multiplicities to decrease, mainly however in the range of $Z_{\text {bound }} / Z_{\text {proj }}>0.5$, because more neutrons are found bound in fragments in this case (cf. Fig. 3). As for the fragment $\langle N\rangle / Z$, the effect is larger for the more neutron-rich system.

For large $Z_{\text {bound }}$, the experimental neutron multiplicities are smaller than the predictions obtained with the standard coefficient $\gamma=25 \mathrm{MeV}$, consistent with the requirement of a reduced symmetry term following from the analysis of the measured isotope distributions of intermediatemass fragments. It is less evident in the case of the neutron-poor ${ }^{124} \mathrm{La}$ for which the sensitivity is small. It is also clear that the neutron multiplicities are overall more uncertain than the precisely measured fragment data. The observed consistency is, nevertheless, rather encouraging.

The yield of neutrons in the range of $Z_{\text {bound }} / Z_{\text {proj }}<0.5$ is not as well reproduced by the present calculations, independently of the chosen coefficient $\gamma$. Several possible reasons can account for this discrepancy. The assumption of an equilibrated spectator source made in the SMM is very successful for fragments, as demonstrated repeatedly and as supported by the rapidity distributions measured for intermediate mass fragments [38]. It is less evident for light particles, as shown for helium isotopes in the same reference. In particular, in the more central collisions with smaller $Z_{\text {bound }}$, a significant tail of helium particles extends to small rapidities, possibly representing a component of preequilibrium emissions or simply the region of overlap with the fireball domain. This is similar for neutrons, apparently, and not accounted for in the calculations with parameters adjusted to best reproduce the yields and correlations of the observed projectile fragments.

\section{Conclusions and outlook}

The emission of neutrons in the fragmentation of excited Sn and La projectiles has been studied with the Large Neutron Detector LAND positioned forward of the ALADIN spectrometer at SIS. The spectator source of neutrons, centered near projectile rapidity, has been identified and its multiplicities and effective temperatures have been determined. The measured mean multiplicities with maximum values of 9 to 12 reflect the neutron richness of the initial projectile, while the temperatures ranging from 3 to $4 \mathrm{MeV}$ are insensitive to it. Calculations with the Statistical Multifragmentation Model show that the neutron multiplicity depends on the strength of the symmetry-term coefficient used in the calculations for the liquid-drop description of fragments. From the study of the fragment isotope distributions measured in the same experiment, this was expected. The experimental multiplicities are in better agreement with the predictions obtained with a reduced symmetry term and thus support the previously reached conclusion [20]. The uncertainties are large, however, because of the extrapolation and corrections needed for the determination of absolute multiplicities.

Besides multiplicities, also the dynamical properties of neutrons in heavy-ion collisions have been found useful for investigating the strength of the nuclear symmetry energy at densities away from normal nuclear density [39]. A value for the strength of the symmetry term at suprasaturation densities has very recently been derived from the comparison of neutron flows with that of charged particles in $\mathrm{Au}+\mathrm{Au}$ reactions at $400 \mathrm{MeV} /$ nucleon [40]. Further results in this direction may be expected from more precise neutron measurements in similar reactions. 


\section{References}

[1] for a review, see P. Nolan and P. Twin, Ann. Rev. Nucl. Part. Sci. 38 (1988) 533.

[2] R. J. Charity, Phys. Rev. C 58 (1998) 1073.

[3] T. Enqvist et al., Nucl. Phys. A 658 (1999) 47.

[4] K. Sümmerer and B. Blank, Phys. Rev. C 61 (2000) 034607.

[5] M. V. Ricciardi et al., Nucl. Phys. A 733 (2004) 299.

[6] P. Napolitani et al., Phys. Rev. C 70 (2004) 054607.

[7] C. Sfienti et al., Phys. Rev. Lett. 102 (2009) 152701.

[8] W. Trautmann, Nucl. Phys. A 787 (2007) 575c.

[9] Dynamics and Thermodynamics with Nuclear Degrees of Freedom, ed. by Ph. Chomaz, F. Gulminelli, W. Trautmann, and S. J. Yennello (Springer, Berlin/Heidelberg/New York 2006); Eur. Phys. J. A 30 (2006) 1.

[10] D. H. E. Gross, Rep. Prog. Phys. 53 (1990) 605.

[11] J. P. Bondorf et al., Phys. Rep. 257 (1995) 133.

[12] A. S. Botvina et al., Nucl. Phys. A 584 (1995) 737.

[13] M. D'Agostino et al., Phys. Lett. B 371 (1996) 175.

[14] M. D’Agostino et al., Nucl. Phys. A 650 (1999) 329.

[15] R. P. Scharenberg et al., Phys. Rev. C 64 (2001) 054602.

[16] N. Bellaize et al., Nucl. Phys. A 709 (2002) 367.

[17] S. P. Avdeyev et al., Nucl. Phys. A 709 (2002) 392.

[18] C. Ishizuka, A. Ohnishi, and K. Sumiyoshi, Nucl. Phys. A 723 (2003) 517.

[19] A. S. Botvina and I. N. Mishustin, Phys. Lett. B 584 (2004) 233; Nucl. Phys. A 843 (2010) 98.

[20] R. Ogul et al., Phys. Rev. C 83 (2011) 024608.

[21] Th. Blaich et al., Nucl. Instrum. Methods Phys. Res. A 314 (1992) 136.

[22] J. Pochodzalla et al., Phys. Rev. Lett. 75 (1995) 1040.

[23] G. J. Kunde et al., Phys. Rev. Lett. 77 (1996) 2897.

[24] L. G. Sobotka et al., Phys. Rev. C 62 (2000) 031603(R).

[25] D. Thériault et al., Phys. Rev. C 71 (2005) 014610.

[26] M. A. Famiano et al., Phys. Rev. Lett. 97 (2006) 052701.

[27] J. Wang et al., Phys. Rev. C 75 (2007) 014604.

[28] V. E. Viola and R. Bougault, Eur. Phys. J. A 30 (2006) 215.

[29] C. A. Ogilvie et al., Phys. Rev. Lett. 67 (1991) 1214.

[30] P. Kreutz et al., Nucl. Phys. A 556 (1993) 672.

[31] J. A. Hauger et al., Phys. Rev. C 62 (2000) 024616.

[32] A. Le Fèvre et al., Phys. Rev. Lett. 94 (2005) 162701.

[33] A. S. Botvina and I. N. Mishustin, Phys. Rev. C 72 (2005) 048801.

[34] J. Iglio et al., Phys. Rev. C 74 (2006) 024605.

[35] G. A. Souliotis et al. Phys. Rev. C 75 (2007) 011601(R).

[36] S. Hudan et al., Phys. Rev. C 80 (2009) 064611.

[37] D. Henzlova et al., J. Phys. G 37 (2010) 085010.

[38] A. Schüttauf et al., Nucl. Phys. A 607 (1996) 457.

[39] for a recent review, see Bao-An Li, Lie-Wen Chen, and Che Ming Ko, Phys. Rep. 464 (2008) 113.

[40] P. Russotto et al. Phys. Lett. B 697 (2011) 471. 